\title{
Atypical Melanoma Misdiagnosed as Viral Meningoencephalitis: A Case Report
}

\author{
Weijun Tang ${ }^{1}$ and Lirong $\mathrm{Wu}^{2 *}$ \\ ${ }^{1}$ Department of Blood Cancers, Jiangjin Central Hospital of Chongqing, China \\ ${ }^{2}$ Department of Neurology, Chongqing Traditional Chinese Medicine Hospital, China
}

\begin{abstract}
As a rare disease with diverse clinical symptoms, primary intracranial malignant melanoma is usually misdiagnosed. We describe a patient who presented intracranial hypertension with abnormal signals in the left temporal lobe. The result of cerebrospinal fluid (CSF) analysis was consistent with the signs of viral meningoencephalitis, so antiviral and nutritional support was given, his headache relieved with normal intracranial pressure. The symptoms of the patients were repeated, and the left temporal lobe lesion was unknown. Surgical biopsy confirmed it was malignant melanoma lastly. This case highlights the brain biopsy in facilitating a highly accurate diagnosis rate.
\end{abstract}

KEYWORDS: Melanoma; Viral meningoencephalitis; Biopsy

ABBREVIATIONS: CT: Computerized Tomographical; MRI: Magnetic Resonance Imaging MRA: Magnetic Resonance Angiography; CSF: Cerebrospinal Fluid; DWI: Diffusion Weighted Imaging; MRS: Magnetic Resonance Spectroscopy; CNS: Central Nervous System

\section{INTRODUCTION}

Melanoma, also known as malignant melanoma, is a type of malignant tumor derived from melanocytes. It is commonly seen in the skin and is also found in the mucosa, eye choroid and other parts of the body. It is the most malignant tumor of skin tumors with a trend of distant metastasis. Due to different melanin levels in the tumor, the presence in magnetic resonance is diversified, and the rate of misdiagnosis is extremely high. Now, we recommend a case of atypical melanoma misdiagnosed as viral meningoencephalitis in our department.

\section{CASE PRESENTATION}

A 21-year-old man was admitted to our hospital because of headache and limb twitching. 2 months ago, the patient was treated in community hospital due to the occurrence of headache and vomiting. The head CT scan showed a slightly high density in the left temporal lobe (images unavailable), and the lumbar puncture showed intracranial pressure is $280 \mathrm{~mm} \mathrm{H} 2 \mathrm{O}$. Routine biochemical of cerebrospinal fluid (CSF) showed white blood cells $30 \times 106 / \mathrm{L}$, monocytes $25 \times 106 / \mathrm{L}$, and multinucleated cells $5 \times 106 / \mathrm{L}$. The man was diagnosed as viral meningoencephalitis and cerebral hemorrhage, so antiviral and nutritional support was given. After two weeks of treatment, his headache and vomiting relieved, with normal intracranial pressure. Nevertheless, the left temporal lobe lesions of the head CT did not improve (images unavailable). Unfortunately, one week later, the patient suffered from a headache again, and he was treated in another hospital. The brain MRI and MRA were examined, and the result is left temporal lobe hemorrhage with the abnormal spot signal, which was consistent with the acute infarction signal, and the MRA was normal (images unavailable). The headache alleviated once again after symptomatic treatment. One month ago, however, the patient had a headache again with stiff neck. He visited the community hospital again and reviewed the lumbar puncture. It was regret that intracranial pressure exceeded $300 \mathrm{~mm}$ H2O. CSF analysis was negative for primary central nervous system (CNS) infections, with normal cell count, glucose, protein and chlorine within reference ranges. At 4 days' interval, the patient had seizures and came to our hospital. The

Quick Response Code:

Address for correspondence: Lirong Wu, Department of Neurology, Chongqing Traditional Chinese Medicine Hospital, China

Received: February 18, $2021 \quad$ Published: February 26, 2021

How to cite this article: Weijun T, Lirong W. Atypical Melanoma Misdiagnosed as Viral Meningoencephalitis: A Case Report. 2021-3(1) OAJBS.ID.000258. DOI: 10.38125/OAJBS.000258 
physical examination showed that the neck resistance was positive, with no other positive signs. Brain MRI showed hyperintensity on the T2-weighted imaging of left temporal lobe and hyperintense FLAIR sequence (Figure 1A). There was mild enhancement zone in the left temporal lobe under enhanced scanning (Figure 1B-C). Dynamic EEG, TCD, blood routine, urine routine, stool routine, liver function, kidney function, blood sugar, erythrocyte sedimentation rate, and thyroid function were normal. Cytomegalovirus and herpes simplex virus antibody IgG $(+)$, and routine biochemical of CSF were normal, and at the same time, Indian ink staining, acid-fast staining, and gram staining of CSF were all negative. His headache relieved once again. The symptoms of the patients were repeated, and the left temporal lobe lesion was unknown. Hence, a transcranial biopsy was performed. The left frontal arachnoid was visible during surgery. In the lower cavity, there were a large number of dark brown lesions, which spread to the subarachnoid space (Figure 2A), and the posterior lesion of the temporal lobe was cut. A large number of black tumors in the brain tissue and arachnoid were observed, and the tumors extensively invaded into the arachnoid, and worsen than that, some tumors are all black. Intraoperative frozen chip results showed most of the samples were brain tissue with small amount of atypical cells mixed, which indicated malignant tumors, and HE staining results revealed malignant tumors (Figure 2B). Hence, a diagnosis of malignant melanoma was made soon after the patient underwent scrupulous ophthalmology and dermatology examinations, as well as chest CT and abdominal CT, which ruled out any other extracranial lesion.
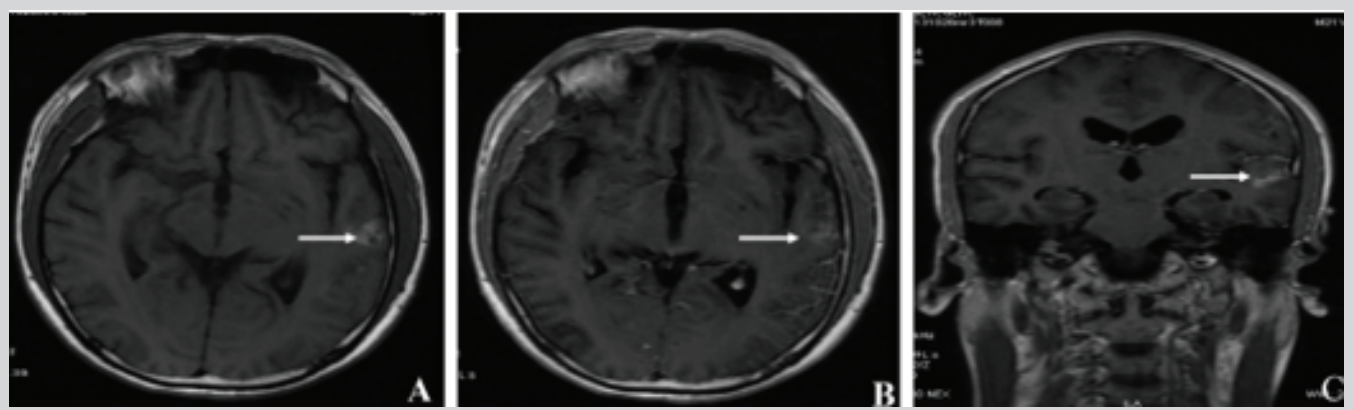

Figure 1: MRI findings. T2 fluid-attenuated inversion recovery [FLAIR](A) and enhanced scanning (B-C) of the patient, demonstrating abnormal signals in the left temporal lobe (arrows).
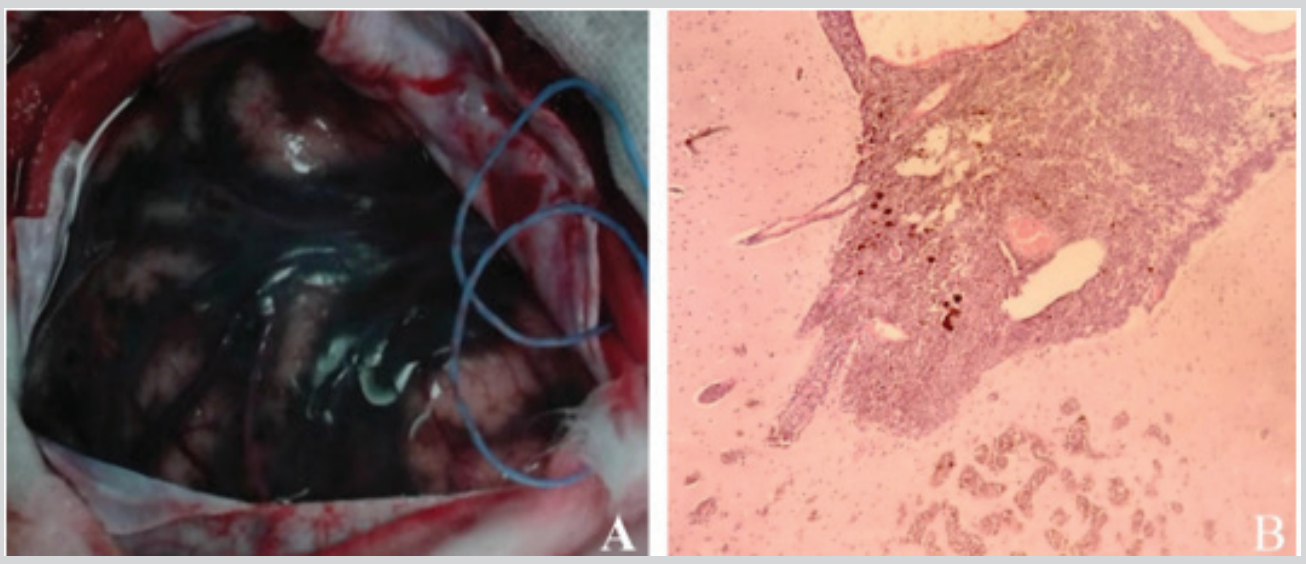

Figure 2: Surgery finding and histology. there were a large number of dark brown lesions in the surgery(A). HE staining results revealed malignant tumors (B).

\section{DISCUSSION}

The melanocytic tumors of central nervous system tumors are divided into meningeal melanocytosis, meningeal melanocytoma, meningeal melanoma and meningeal melanomatosis, of which the latter two are classified as malignant lesions [1]. CNS melanoma tumors are mostly transferred from other systems and ranks third among the most common brain metastases tumors [2]. Melanoma originated from the CNS is very rare, accounting for only $1 \%$ of all melanomas, $0.07 \%$ of brain tumors [3-5], and CNS melanoma can be transferred to other sites. Wills [6] proposed three basic indications to diagnose primary central nervous system melanoma: (1) no melanoma found in the skin and eyeball; (2) no previous melanoma resection; (3) no melanoma metastasis found in the visceral organs. Intracranial melanoma can be roughly divided into three categories: 1) primary melanoma; 2) metastatic melanoma;
3) melanin type of various central nervous system tumors [7-8]. Primary melanoma occurs mostly in the pia mater and arachnoid membrane, while on the contrary, metastatic melanoma occurs mostly at the junction of the cortex and medulla, and less invades the meninges [9]. Primary melanoma is more commonly seen in single lesion, and metastasis is commonly seen in multiple lesions. Two essential factors are required to make a definite diagnosis of primary intracranial melanoma. First, the pathological results of histology must be obtained, regardless of your histological specimen is obtained by endoscopy or surgery. Second, a large number of clinical and radiological examinations are needed to exclude other central system metastases [10]. Some scholars have found that high-dose radiation therapy and surgical treatment are comparable in the treatment of primary intracranial melanoma [11]. The effect of chemotherapy on primary central nervous system melanoma is not ideal, compared with immunotherapy on metastatic melanoma 
[12-13]. The prognosis of primary central nervous system melanoma is generally better than that of metastatic melanoma. The average life span of primary central nervous system melanoma is up to 4 years after diagnosis, while that of metastatic melanoma is generally less than 6 months [10]. As a variant of malignant melanoma, meningeal melanoma, an insubstantial tumor, is mainly located in the skull base meninx vasculosa and can invade into the spinal canal. The signal of melanoma on $\mathrm{T} 1$ is positively correlated with the melanin contents, and different melanin contents will cause different MRI signal performances [14]. Some scholars find that the molecular diagnosis of melanoma is extremely important [15]. It is usually stained positively with HMB-45 and S-100. S-100 possesses the highest sensitivity but poor specificity. Melan-A and HMB45 are of higher specificity, suggesting that melanin is active [16]. So far, however, there are no guidelines or recommendations for the diagnosis of primary melanoma [17]. More and more studies in recent years have shown that DWI, MRI dynamic enhanced scanning, MRS and perfusion imaging can be beneficial to the diagnosis of intracranial melanoma. Unfortunately, these methods failed to identify the disease, as they only indicated the location and property of the lesion, but finally, the brain tissue biopsy succeeded in making a definite diagnosis.

This case highlights the significance of pathological biopsy in clinical diagnosis. The abnormal intracranial signals can occur in viral meningoencephalitis, such as herpes simplex virus. Initially, we recommended brain tissue biopsy repeatedly to the patient who was misdiagnosed as viral meningoencephalitis and suffered from poor treatment. However, brain tissue biopsy is not widely accepted by Chinese, Chinese rural people in particular. Ultimately, brain tissue biopsy confirmed the diagnosis.

\section{CONCLUSION}

During the course of diagnosis, clinicians should thoroughly get wise to the patients' past clinical history and use multiple auxiliary checks without the influence of traditional beliefs.

\section{CONFLICT OF INTEREST}

No conflict of interest exists in the submission of this manuscript. I would like to declare on behalf of my co-authors that the work described is original research that has not been published previously, and not under consideration for publication elsewhere, in whole or in part. All the authors listed have approved the manuscript that is enclosed.

\section{ETHICS APPROVAL AND CONSENT TO PARTICIPATE}

The procurement of this study was obtained with written patient-informed consent and approved by the Institutional Ethics Committee Faculty of Medicine at Chongqing Traditional Chinese Medicine Hospital.

\section{CONSENT TO PUBLISH}

Written consent has been obtained from the patient for the publication of this case report and all accompanying images.

\section{AVAILABILITY OF DATA AND MATERIALS}

The datasets supporting the conclusions of this article are included within the article.

\section{AUTHOR'S CONTRIBUTION}

WJ and LR designed the paper, LR collect the data and followedup the patient, WJ and LR drafted the manuscript, WJ and LR edited the manuscript. The author (s) read and approved the final manuscript.

\section{REFERENCES}

1. Louis DN, Perry A, Reifenberger G, Von Deimling A, Figarella BD, et al. (2016) The 2016 world health organization classification of tumors of the central nervous system: a summary. Acta Neuropathol 131(6): 803820.

2. Hirota K, Yoshimura C, Kubo O, Kasuya H (2017) Primary intracranial malignant melanoma with extracranial metastasis. J Korean Neurosurg Soc 60(1): 98-101.

3. Balakrishnan R, Porag R, Asif DS, Satter AMR, Taufiq M, et al. (2015) Primary intracranial melanoma with early leptomeningeal spread: A case report and treatment options available. Case Rep Oncol Med 2015: 293802.

4. Greco CS, Soffietti R, Bradac GB, Boldorini R (2001) Primitive cerebral melanoma: case report and review of the literature. Surg Neurol 55(3): 163-168.

5. Azimi P, Mohmmadi HR, Refiezadeh M (2012) Primary pineal melanoma presenting with leptomeningeal spreading in a 22 -year-old woman: a case report. J Med Case Rep 27(6): 165.

6. Willis RA (1965) The Hamartomatous syndromes: their clinical, pathological and fundamental aspects. Med J Aust 1(23): 827-833

7. Liubinas SV, Maartens N, Drummond KJ (2010) Primary melanocytic neoplasms of the central nervous system. J Clin Neurosci 17(10): 12271232.

8. Xie ZY, Hsieh KLC, Tsang YM, Cheung WK, Hsieh CH, et al. (2014) Primary leptomeningeal melanoma. J Clin Neurosci 21(6): 1051-1052.

9. Zhang N, Liu ZJ, Li G, Xiao B, Liu YH, et al. (2007) Clinical and pathologic features of melanocytic lesion of the central nervous system. Zhong Nan Da Xue Xue Bao Yi Xue Ban 32(4): 713-717.

10. Todeschi J, Chibbaro S, Clavier JB, Lhermitte B, Goichot B, et al. (2016) An unusual pituitary stalk lesion: What is the place of surgery? Neurochirurgie 62(6): 339-343.

11. Classen J, Hehr T, Paulus W, Plate K, Bamberg M, et al. (2002) Suprasellar melanocytoma: a case of primary radiotherapy and review of the literature. J Neurooncol 58(1): 39-46.

12. Biswas A, Chaudhari PB, Sujith KM, Sigamani E, Sharma MC, et al. (2015) Primary pineal malignant melanoma - illustrated review. Turk Neurosurg 25(2): 201-209.

13. Copeland DD, Sink JD, Seigler HF (1980) Primary intracranial melanoma presenting as a suprasellar tumor. Neurosurgery 6(5): 542-545.

14. Gaviani P, Mullins ME, Braga TA, Hedley WET, Halpern EF, et al. (2006) Improved detection of metastatic melanoma by $\mathrm{T} 2 *$-weighted imaging. AJNR Am J Neuroradiol 27(3): 605-608.

15. Trinh V, Medina FR, Taylor CL, Yonas H, Chohan MO, et al. (2014) Primary melanocytic tumors of the central nervous system: Report of two cases and review of literature. Surg Neurol Int 13(5): 147.

16. Shang JX, Zhang D, Zhang JT, Zhao JZ, Zhang Y, et al. (2013) Melanocytic neoplasms of central nervous system analysis. Zhonghua Yi Xue Za Zhi 93(1): 34-36

17. Wadasadawala T, Trivedi S, Gupta T, Epari S, Jalali R (2010) The diagnostic dilemma of primary central nervous system melanoma. J Clin Neurosci 17(8): 1014-1017. 